\title{
Consumo de tabaco y alcohol entre los estudiantes de medicina de la Pontificia U niversidad Católica de Chile
}

\author{
María Inés Romero ${ }^{1}$, Jaime Santander ${ }^{2}$, \\ Mario Javier Hitschfeld ${ }^{3}$, Marcela Labbéé, Viviana Zamora ${ }^{5 b}$. \\ Smoking and alcohol drinking \\ among medical students at the \\ Pontificia Universidad Católica \\ de Chile
}

Background: Tobacco and alcohol consumption are public health problems, generally starting in adolescence. Medical students are not an exception. Aim: To determine the characteristics of tobacco and alcohol use among medical students at the Pontificia Universidad Católica de Chile and their association with gender, career level and mental health. Material and methods: A questionnaire to evaluate substance use was applied along with Goldberg Health Questionnaire (GHQ-12), as a mental health risk predictor, to all medical students in November 2005. Results: The survey was completed by 569 of 775 students (74\%). Current smokers were $23.7 \%$, with the lowest figure, $13.5 \%$ in sixth year, and the highest, $40.5 \%$ in seventh year $(p<0.01)$. Thirty one percent of students with an score of five and over in the GHQ-12 were smokers, compared to 19\% among those with a lower score. Daily smokers were $40 \%$ of the current smokers. Seventy four percent of students consumed alcohol during the last month. No association with sex or GHQ-12 was observed. The lowest alcohol consumption rate was observed in second year, and the highest in sixth year (66\% and $89.2 \%$, respectively, $p<0.01$ ). Fifty three percent of men and $26 \%$ of women drank three or more drinks in any given day $(p<0.01)$. Sixty three percent of men and $81 \%$ of women never drank more than five drinks in one day, during the last month $(p<0.01)$. Conclusions: Our medical students smoke less than Chilean youth but more than medical students of countries such as the USA. They drink less than Anglo-Saxon medical students but more than Chilean youth. Male consumption is greater than that of women. Smoking and alcohol drinking are mutually associated (Rev Méd Chile 2009; 137: 361-8).

(Key words: Alcohol drinking; Smoking; Students, medical)

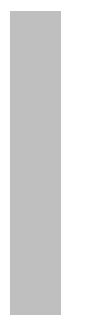

\footnotetext{
Recibido el 4 de junio, 2008. Aceptado el 1 de diciembre, 2008.

Este trabajo no recibió financiamiento. Su realización contó con apoyo logístico por parte de la Dirección de Pregrado y el Departamento de Salud Pública de la Facultad de Medicina de la Pontificia Universidad Católica de Chile.

${ }^{1}$ Dirección de Pregrado y Departamento de Salud Pública, ${ }^{2}$ Departamento de Psiquiatría, ${ }^{3}$ Departamento de Salud Pública, ${ }^{4}$ Escuela de Medicina, ${ }^{5}$ Dirección de Pregrado, Facultad de Medicina, Pontificia Universidad Católica de Chile. Santiago de Chile.

${ }^{a}$ Interna de Medicina, Pontificia Universidad Católica de Chile

${ }^{\mathrm{b}}$ Asistente Social
}

Correspondencia a: Dra. María Inés Romero. Marcoleta 434,

Santiago, 833-0073, Chile. Fono: 3543038. Fax: 6331840.

E mail: mromeros@uc.cl 
$F^{4}$ umar cigarrillos y beber alcohol son hábitos presentes en gran parte de la población y, dadas las características del consumo, se constituyen en problemas de salud pública.

Estudios realizados en Chile por diversos autores muestran que se inician generalmente en la adolescencia, en edades progresivamente más tempranas y en relación a un entorno de consumidores $^{1-8}$. Las escuelas de medicina no están ajenas a este fenómeno, existiendo numerosa bibliografía internacional que da cuenta de consumos de magnitud variable, generalmente alto ${ }^{9-21}$. En el caso del alcohol, se han descrito al estrés y las presiones académicas, elementos propios de la carrera de medicina, como factores que esgrimen los estudiantes para consumirlo $9,11,13,19$. Dado que la información nacional es muy escasa, y con el fin de aproximarse al conocimiento de la realidad en estudiantes de medicina chilenos, se realizó el presente estudio.

La medicina debe enfrentar los fenómenos del tabaquismo y del abuso de alcohol como problemas de salud pública cruciales. Si el profesional de salud es consumidor de tabaco o alcohol, no tendrá motivación suficiente para prevenir el inicio del consumo en otras personas ni para movilizar cambios de conducta y su intervención será menos exitosa 22,23 . A esto se suma el importante efecto de modelaje en el inicio, mantención y cesación de consumos por parte de figuras significativas, por lo que el médico debiera ser un ejemplo para su comunidad en cuanto a estilos de vida saludable.

A fin de conocer cuál es la frecuencia y características del uso de tabaco y alcohol, se realizó una encuesta de consumo en estudiantes de primero a séptimo año de medicina de la Pontificia Universidad Católica de Chile (PUC). Los objetivos planteados fueron: cuantificar la frecuencia del consumo de tabaco y alcohol; caracterizar el consumo de estas sustancias según cantidad y periodicidad del consumo; caracterizar el consumo según sexo y nivel de la carrera, y según presencia de predictores de riesgo de salud mental.

\section{MATERIAL Y MÉTODO}

El presente estudio corresponde a un diseño de prevalencia realizado en la totalidad de los estu- diantes de medicina de la PUC en noviembre de 2005. La encuesta aplicada se componía de dos instrumentos: Encuesta de consumo de sustancias, usada y validada por el Consejo Nacional para el Control del Consumo de Estupefacientes (CONACE), aplicada regularmente a jóvenes en edad escolar y universitaria de Chile cada 2 años y Cuestionario de Salud General abreviado de Goldberg (GHQ12) ${ }^{24}$ considerado un predictor de riesgo en salud mental, usado ampliamente en todo el mundo, validado en nuestro medio ${ }^{25}$ y utilizado previamente en nuestra Escuela ${ }^{26}$. Esta publicación centra su análisis en el módulo de consumo de sustancias con sus variables de tabaco y alcohol.

La encuesta fue entregada a todos los estudiantes de cada curso para su autoadministración, solicitando colaboración voluntaria, firma de consentimiento informado y asegurando anonimato y confidencialidad. El proceso fue supervisado por el equipo investigador. Las variables dependientes fueron consumo de tabaco y alcohol. Las variables independientes fueron sexo, curso y predictor de riesgo en salud mental.

El consumo de tabaco fue medido en prevalencia de vida (alguna vez en su vida), actual y número diario de cigarrillos entre los que consumen diariamente. El consumo de alcohol fue medido en prevalencia de vida, anual (en el último año) y mensual (en el último mes) y, en este último grupo, frecuencia de consumo dentro del mes, intensidad de consumo en un día habitual y número de días dentro de un mes en que se consume excesivamente, definido como consumir más de 5 tragos en una sesión (1 trago $=13 \mathrm{~g}$ alcohol). El predictor de riesgo en salud mental fue definido como puntaje igual o superior a 5 en el GHQ-12, denominándose "GHQ-12 positivo".

Los datos se procesaron en programas Epiinfo y SPSS, aplicando las pruebas de significación estadística pertinentes: diferencia de proporciones y chi cuadrado para estudiar la asociación de los consumos según las variables elegidas.

\section{Resultados}

La encuesta fue respondida por 569 de 775 estudiantes (74\%). La tasa de respuestas superó $90 \%$ en los cursos de $1^{\circ}$ a $4^{\circ}$, alcanzando un 
mínimo de 34\% en los cursos del internado. La distribución según sexo de los respondentes representa la distribución del universo, con 56\% de varones (Tabla 1). La edad promedio fue 21,4 años (DE 2,1; rango 18 a 30 años).

Treinta y nueve por ciento de los estudiantes presentó un GHQ-12 positivo, con un máximo de $52 \%$ en primer año descendiendo linealmente hasta un mínimo de $14 \%$ en quinto año, asociación estadísticamente significativa ( $\mathrm{p}<0,0001)$ (Figura 1).

Tabaco. El 53,6\% de los alumnos ha consumido tabaco alguna vez. El $23,7 \%$ consume tabaco actualmente, sin diferencias significativas entre sexos (Figura 2).

El consumo actual por curso aumenta de primero a cuarto año, disminuye al mínimo en sexto año (13,5\%), alcanzando el máximo en séptimo (40,5\%), asociación significativa ( $<<0,0004)$ (Figura 3).

El consumo actual de tabaco alcanza 31\% en los estudiantes con GHQ-12 positivo y 19\% en aquellos con GHQ-12 negativo, asociación significativa ( $\mathrm{p}<0,007)$.

De los fumadores, $40 \%$ fuma todos los días, mostrándose en Tabla 2 su distribución según número de cigarrillos diarios consumidos, sin asociación significativa con sexo, curso ni GHQ-12.

Tabla 1. D istribución de estudiantes de medicina respondentes a una encuesta sobre consumo de tabaco y alcohol, según curso y sexo

\begin{tabular}{|c|c|c|c|c|c|c|}
\hline \multirow[t]{2}{*}{ Curso } & \multicolumn{2}{|c|}{ Hombres } & \multicolumn{2}{|c|}{ Mujeres } & \multicolumn{2}{|c|}{ Total } \\
\hline & $\mathbf{n}$ & $(\%)$ & $\mathrm{n}$ & $(\%)$ & $\mathbf{n}$ & $(\%)$ \\
\hline $1^{\text {음 }}$ & 63 & (62) & 39 & (38) & 102 & (100) \\
\hline $2^{\mathrm{o}}$ & 68 & (60) & 46 & (40) & 114 & (100) \\
\hline $3^{\circ}$ & 64 & (56) & 51 & (44) & 115 & (100) \\
\hline $4^{\circ}$ & 48 & (48) & 53 & (52) & 101 & (100) \\
\hline $5^{\circ}$ & 34 & (54) & 29 & (46) & 63 & (100) \\
\hline $6^{\circ}$ & 17 & (46) & 20 & (54) & 37 & (100) \\
\hline 70 & 24 & (65) & 13 & (35) & 37 & (100) \\
\hline Total & 318 & $(56)$ & 251 & (44) & 569 & (100) \\
\hline
\end{tabular}

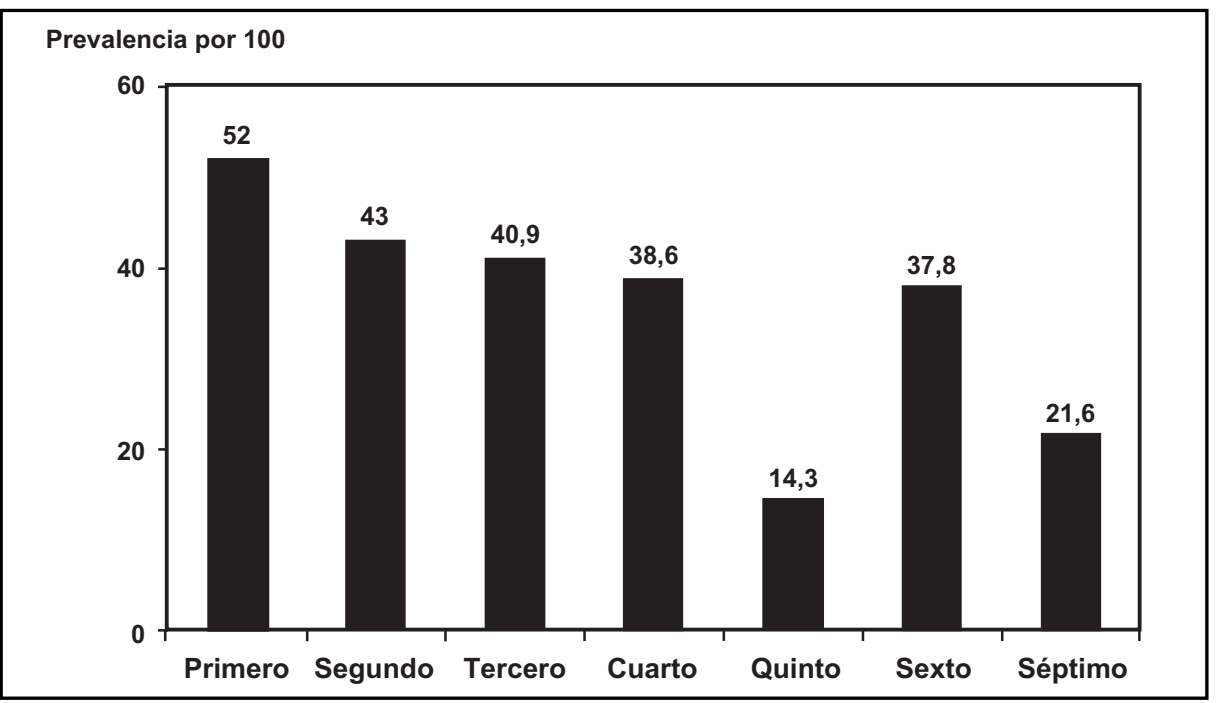

Figura 1. Prevalencia de GHQ-12 positivo (puntaje igual o superior a 5), por 100 estudiantes, según curso. 


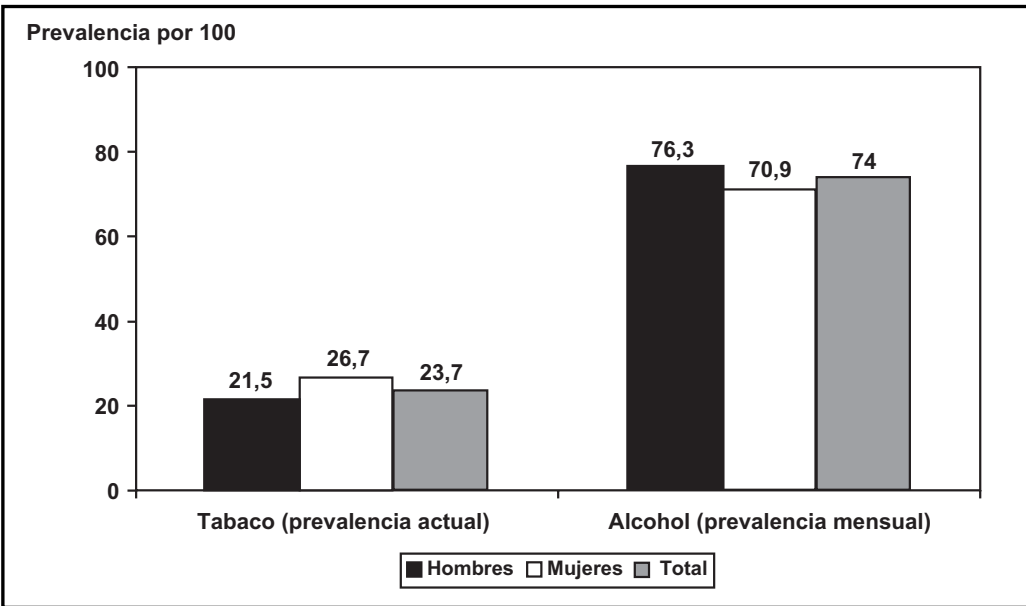

Figura 2. Prevalencia de consumo actual de tabaco y último mes de alcohol, por 100 estudiantes, según sexo.

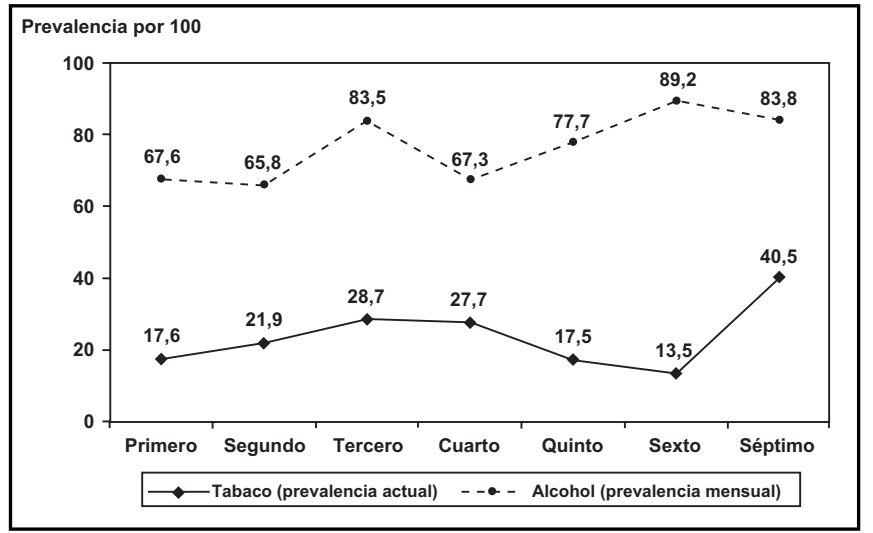

Figura 3. Prevalencia de consumo actual de tabaco y último mes de alcohol, por 100 estudiantes, según curso.

Noventa y tres por ciento de los fumadores y $68 \%$ de los no fumadores ha bebido alcohol durante el último mes, asociación significativa $(\mathrm{p}<0,0001)$.

Alcohol. El 92\% de los estudiantes ha consumido alcohol alguna vez en su vida, $89 \%$ ha consumido en el último año y $74 \%$ en el último mes.

No se encontraron asociaciones significativas en el consumo de vida, último año y último mes con sexo y riesgo de salud mental. Tampoco se encontró asociación para consumo de vida y último año con nivel de la carrera (curso).

Los resultados más relevantes se encontraron para el consumo durante el último mes. Las cifras más bajas (alrededor de 66\%) se observaron en primer, segundo y cuarto año; cifras sobre $80 \%$ se alcanzaron en tercer año e internados, con un máximo de $89 \%$ en internado de sexto. La asociación es significativa $(\mathrm{p}<0,004)$ (Figura 3 ).

De aquellos que beben, 1,1\% lo hace todos los días, $40,3 \%$ los fines de semana, 9,1\% algunos días de la semana y $49,6 \%$ ocasionalmente en el mes, sin asociación significativa entre sexos, curso, ni tests de salud mental.

La cantidad de consumo en un día habitual (Figura 4) muestra asociación significativa con sexo, mayor en hombres ( $p<0,0001)$, sin diferencias por curso, ni GHQ-12.

La intensidad de consumo (Figura 5), muestra la cantidad de días que han consumido más de cinco 
Tabla 2. D istribución porcentual de consumo de cigarrillos diarios entre estudiantes que fuman todos los días

\begin{tabular}{|cc|}
\hline $\begin{array}{c}\text { Número de } \\
\text { cigarrillos diarios }\end{array}$ & $\begin{array}{c}\text { Porcentaje } \\
\text { de estudiantes }\end{array}$ \\
\hline 1 & 30,7 \\
2 & 16,1 \\
3 & 9,5 \\
4 & 10,2 \\
5 & 7,3 \\
6 & 1,5 \\
7 & 4,4 \\
8 & 1,5 \\
10 & 9,5 \\
12 & 0,7 \\
15 & 2,9 \\
20 & 5,8 \\
\hline
\end{tabular}

tragos, destacando también el predominio masculino, con asociación significativa $(p<0,003)$ y, nuevamente, sin asociación con curso ni GHQ-12.

En los que han bebido durante el último mes, el 30\%, además, fuma tabaco, mientras que entre aquellos que no han bebido, sólo 6\% consume tabaco, asociación significativa ( $p<0,0001)$.

\section{Discusión}

No existen publicaciones nacionales que estudien el consumo de tabaco y alcohol entre estudiantes de medicina. Curiosamente, hay publicaciones internacionales que también señalan no encontrar estudios similares al de ellos en sus países ${ }^{15}$. Ello representa un desafío para que otras escuelas de medicina chilenas emprendan estudios similares para recoger la realidad nacional y su evolución en el tiempo, hecho esencial para medir el impacto de las medidas que se tomen.

Nuestro estudio muestra que el número de estudiantes de medicina que fuma es menor al de sus pares en general. Aunque nos preocupa que $23,7 \%$ de nuestros estudiantes fuma actualmente, la cifra es más baja que sus pares etarios (58\%) y universitarios $(50 \%)$ chilenos ${ }^{3,6}$. Los factores protectores más importantes, según la literatura internacional, serían la formación médica que reciben, la influencia de sus compañeros y el rol médico como modelo para sus pacientes ${ }^{10,15}$. También

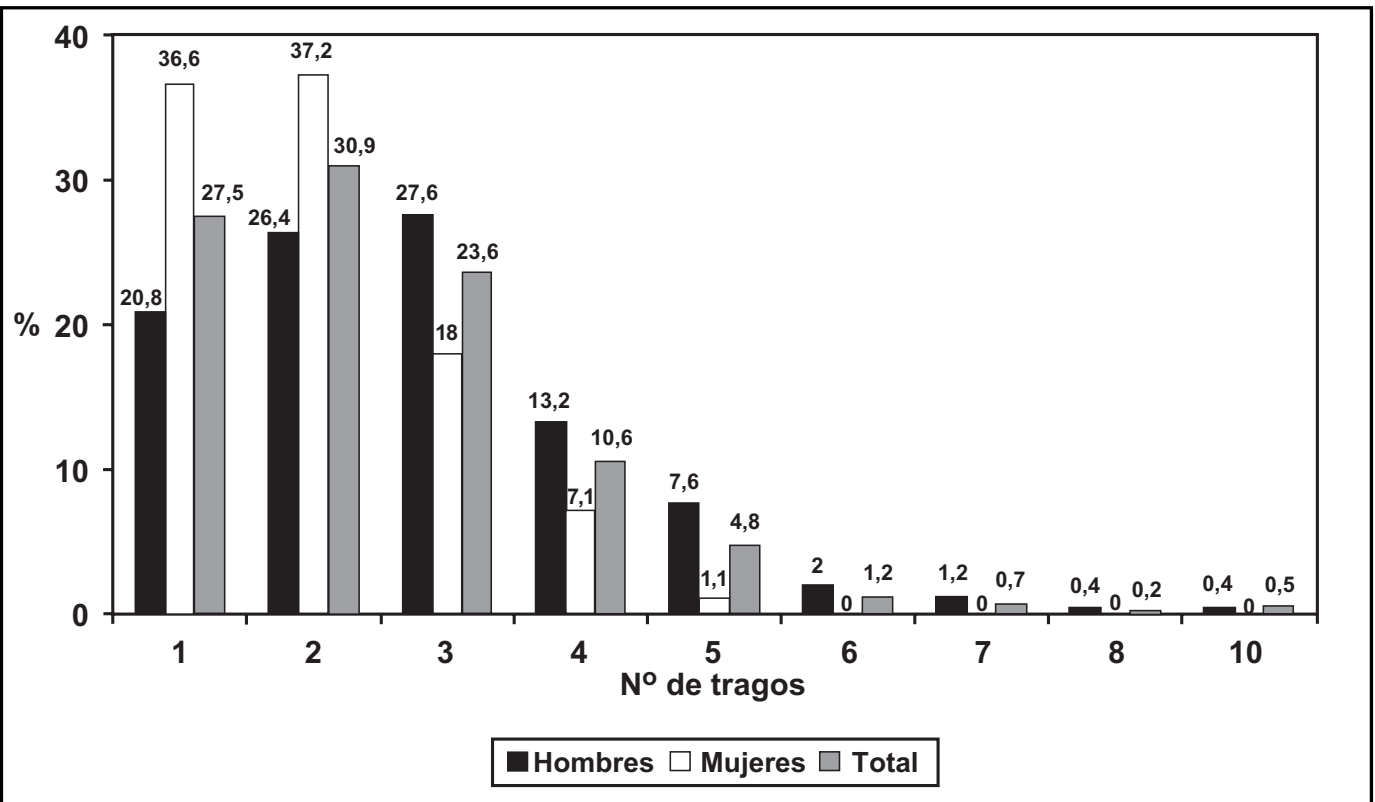

Figura 4. Distribución porcentual de estudiantes que han consumido alcohol durante el último mes, según sexo y número de tragos en un día habitual (1 trago =13 g alcohol). 


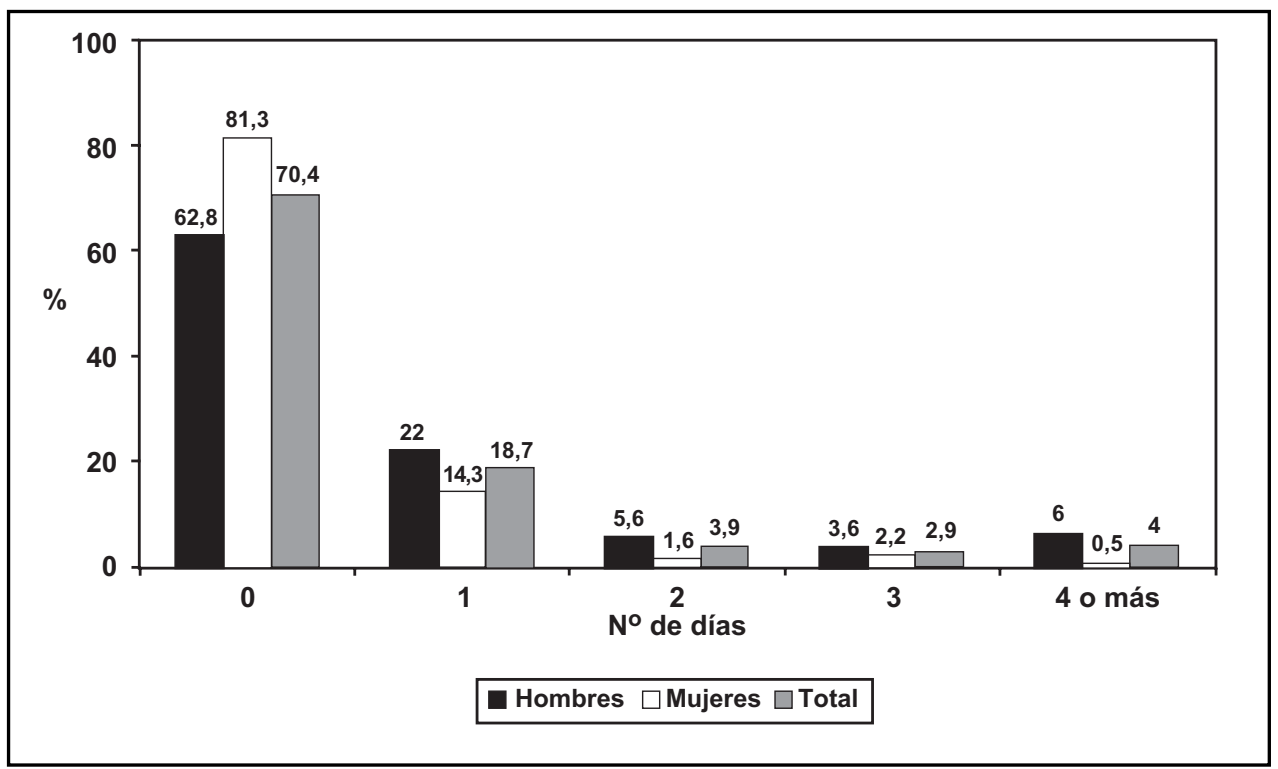

Figura 5. Distribución porcentual de estudiantes que han consumido alcohol durante el último mes, según sexo y número de días que han bebido más de cinco tragos por sesión ( 1 trago =13 g alcohol).

aparece en nuestro estudio la fuerte asociación entre tabaco y alcohol, lo que es preocupante dado que se sabe que la presencia simultánea de consumo de tabaco y alcohol potencia sus efectos negativos.

La ausencia de asociación estadística entre sexo y consumo de tabaco (hombres: 21,5\%; mujeres: 26,7\%), coincide con lo visto en jóvenes chilenos durante los últimos 10 años, en que se ha acortado notoriamente la brecha entre hombres y mujeres, siendo actualmente de 59,7\% y 55\%, respectivamente 7 . Además, esta escasa diferencia entre consumo masculino y femenino no sorprende ya que Chile, dentro del contexto latinoamericano, es el país con menor diferencia entre sexos en el consumo de tabaco ${ }^{27}$.

Comparando estudiantes de medicina, vemos que nuestros estudiantes tienen cifras de consumo de tabaco inferiores o similares a países como Turquía (32,1\%; 37\%), Italia (30\%) y Colombia $(25,9 \%)^{9-11,14,15,18}$, y mayores que Estados Unidos de Norteamérica (EE.UU.) (2\%; 3,3\%). Esta diferencia con EE.UU. es consistente con las altas cifras generales de tabaquismo que muestra nuestro país dentro del contexto internacional y confirman la existencia de una situación preocupante de consumo. Esto se explicaría, en parte, por la insuficiencia de las campañas anti-tabaco, la presencia de publicidad por parte de las tabacaleras y las escasas leyes restrictivas hacia el tabaco ${ }^{11,14}$, hasta el año 2007 cuando entró en completa vigencia la nueva ley de tabacos en nuestro país. Se espera que esta medida tenga un impacto en las cifras, aunque con latencia de algunos años, logrando acercarse a las de países desarrollados que sí tienen, desde hace tiempo, leyes restrictivas al consumo de tabaco.

La asociación entre tabaquismo y curso es consistente con un estudio turco que probó que un factor de riesgo para tabaquismo es ser estudiante de últimos cursos 9 . Aquello nos genera la pregunta: ¿Los médicos fumarán más que los estudiantes de medicina? Otro estudio turco ${ }^{11}$ muestra que los médicos fuman menos que los estudiantes, lo cual nos permitiría postular que, en nuestro estudio, el consumo máximo en séptimo año sería un hecho puntual en una curva descendente de consumo que se iniciaría en quinto año. El incremento del consumo de tabaco en séptimo año podría explicarse por la mayor presión del internado, el pronto egreso y su incertidumbre respecto a inserción laboral y especialización, lo 
que estaría apoyado por la asociación entre GHQ12 positivo y tabaquismo encontradas. Sin embargo, variados estudios internacionales no mostraron asociación entre ansiedad, depresión y tabaquismo 9,11,17,19. Estudios de seguimiento en nuestros estudiantes podrán aclararnos si el incremento en séptimo año es un hecho aislado o responde a condiciones permanentes del estudiante en ese año.

Queda mucho por avanzar en la lucha contra el tabaquismo entre los estudiantes de medicina de nuestra Universidad. Si bien en la comparación nacional la prevalencia es más baja que la de sus pares, al compararse con estudiantes de medicina de países más desarrollados se aprecia el gran margen que queda por mejorar. Incorporar y reforzar la enseñanza ordenada y formal sobre los efectos negativos del tabaco en las Escuelas de Medicina debe ser prioridad, ya que la ausencia de ella perpetúa las altas prevalencias ${ }^{10}$. El claro beneficio para la salud del estudiante de medicina y la necesidad de ser un buen ejemplo para los pacientes, son algunas razones para disminuir el consumo de tabaco ${ }^{10,15}$. Las metas debieran ser alcanzar cifras similares a los países desarrollados $y$, en paralelo, avanzar en que los equipos de salud efectivamente intervengan para que sus pacientes dejen de fumar, algo que no ocurre en la actualidad ${ }^{15}$.

Las cifras de consumo de alcohol entre nuestros estudiantes son preocupantes. Mientras en la población general y en la Educación Superior la prevalencia anual es 59\% y $74 \%$, respectivamente, en nuestros estudiantes es de $89 \%{ }^{6}$. En la comparación internacional la prevalencia mensual en estudiantes turcos, por ejemplo, es de $26 \%$ y $42 \% 9,11$, en tanto la nuestra alcanza a $74 \%$.

Nuestra situación parece mejor si nos comparamos con países anglosajones como EE.UU. y el Reino Unido. El consumo de alcohol entre sus estudiantes de medicina es mayor, oscilando entre $86 \%$ y $95 \% 16-20$, y su intensidad de consumo en un día habitual también es superior ${ }^{16,17,19}$. Elementos culturales y religiosos pueden explicar esta diferencia entre países9, por lo que no nos deben conformar comparaciones favorables frente a países anglosajones.

Nuestro estudio no exploró las razones que dan los bebedores para consumir alcohol, pero podemos asumir que son similares a las expuestas en estudios internacionales, como placer, estrés, presión social y de los pares, aumentar la confianza, hábito y presiones académicas $9,11,13,19$.

Al desglosar por sexo, se ve que no existe diferencia de frecuencia de consumo entre hombres y mujeres, hecho también observado en países como Turquía y el Reino Unido9,16,19. Variados estudios nacionales muestran que los hombres beben más que las mujeres ${ }^{1-3,5,6}$, pero también muestran que desde 1990 aumenta la prevalencia anual de consumo entre mujeres, en tanto se mantiene estable la de los hombres, acercándose cada vez más ambas cifras ${ }^{2}$. Además estudios en jóvenes muestran un patrón similar de consumo en ambos sexos ${ }^{2,5}$. Por ello se puede plantear que nuestra igualdad de frecuencia de consumo entre hombres y mujeres es sólo un adelanto de lo que será la tendencia en el país.

Al analizar la cantidad de consumo en un día habitual, se observa que entre los estudiantes de medicina nacionales y extranjeros, los hombres beben más tragos y tienen mayores cifras de abuso de alcohol que las mujeres, encontrándose este patrón también en la población general2,17,18.

En cuanto a consumo y etapa de la carrera, estudios extranjeros similares muestran que mientras más avanzado en la carrera médica, mayor es el consumo de alcohol ${ }^{19,20}$, lo cual es concordante con lo visto en nuestro estudio. Esta tendencia no guarda relación con estrés o depresión, hecho registrado en variados estudios internacionales y también en el nuestro, a través de la ausencia de relación con GHQ-12 positivo. Sin duda el aumento con la edad es una luz de alerta a favor de la importancia en la prevención de consumo de alcohol a todo nivel en el equipo de salud ya que, según estudios internacionales, el problema entre los médicos puede ser aún mayor ${ }^{11,16,20}$.

Es un gran desafío moderar el consumo de alcohol entre los estudiantes de medicina y disminuir la preocupante cifra de bebedores que ingieren más de cinco tragos en un solo día. Este desafío no es sólo nuestro, es una realidad compartida a nivel internacional y, a diferencia del tabaco, podemos dar ejemplo de que es posible mejorar. En nuestra Universidad existe una política de prevención del uso de drogas ilegales y del abuso de alcohol desde noviembre de 2006 aplicable a todos sus estudiantes, que 
centra su acción en el contexto preventivo (disponible en www.uc.cl), la cual es motivada también por las altas cifras de consumo que se observan

\section{REFERENCIAS}

1. CONACE. Consumo precoz de alcohol y su relación con consumo de marihuana y cocaína. Cuarto estudio nacional de drogas en población general de Chile 2000; Julio 2001.

2. CONACE. Informe sobre uso, abuso y dependencia al alcohol. Quinto estudio nacional de drogas en población general de Chile 2002; Diciembre 2003.

3. CONACE. Consumo de drogas en jóvenes. Sexto estudio nacional de drogas en población general de Chile 2004

4. CONACE. Sexto estudio nacional de drogas en población escolar de Chile de $8^{\circ}$ Básico a $4^{\underline{0}}$ Medio 2005; Informe final.

5. CONACE. Situación del consumo de drogas en mujeres de Chile. Quinto estudio nacional de drogas en población general de Chile 2002; Agosto 2004.

6. CONACE. Informe sobre uso de drogas en estudiantes de la educación superior. Quinto estudio nacional de drogas en población general de Chile 2002; Junio 2003.

7. CONACE. El consumo de cigarrillos en Chile. Estudios nacionales de drogas en población general de Chile 1994-2004. Estudios nacionales de drogas en población escolar de Chile 1995-2003; Mayo 2005.

8. SAPAG J, Florenzano R. Adolescencia: promoción, prevención y atención de salud. Ediciones Universidad Católica, 2002; 201-18.

9. Akvardar Y, Demiral Y, Ergör G, Ergör A, Bilici M, Ozer OA. Substance use in a sample of Turkish medical students. Drug Alcohol Depend 2003; 72: 117-21.

10. Melani AS, Verponziani W, Boccoli E, Trianni GL, Federici A, Amerini R et al. Tobacco smoking habits, attitudes and beliefs among nurse and medical students in Tuscany. Eur J Epidemiol 2000; 16: 607-11.

11. Akvardar Y, Demiral Y, Ergör G, Ergör A. Substance use among medical students and physicians in a medical school in Turkey. Soc Psyquiatry Psychiatr Epidemiol 2004; 39: 502-6.

12. Baus J, Kupek E, Pires M. Prevalence and risk factors associated with drug use among school students, Brazil. Rev Saude Publica 2002; 36: 40-6.

13. De Andrade V, Pereira L, Guerra A, Nicastri S, Malbergier A. Comparative study of drug use among undergraduate students at the University of SaO Paulo, Sao Paulo campus in 1996 and 2001. Rev Bras Psiquiatr 2005; 27: 185-93. hace años en la educación superior. Esta iniciativa, independiente de nuestro estudio, sin duda significa un avance, quedando aún mucho por mejorar.

14. Rosselli D, Rey O, Calderón C, Rodríguez MN. Smoking in Colombian medical schools: The hidden curriculum. Preventive Medicine 2001; 33: 170-4.

15. Patkar A, Hill K, Batra V, Vergare M, Leone F. A comparison of smoking habits among medical and nursing students. Chest 2003; 124: 1415-20.

16. Pickard M, Bates L, Dorian M, Greig H, Saint D. Alcohol and drug use in second-year medical students at the University of Leeds. Medical Education 2000; 34: 148-50.

17. Newbury-Birch D, White M, Kamali F. Factors influencing alcohol and illicit drug use amongst medical students. Drug and Alcohol Dependence 2000; 59: 125-30.

18. Mangus R, Hawkins C, Miller M. Tobacco and alcohol use among 1996 medical school graduates. JAMA 1998; 280: 1192-3

19. Newbury-Birch D, Walshaw D, Kamali F. Drink and drugs: From medical students to doctors. Drug and Alcohol Dependence 2001; 64: 265-70.

20. Mcauliffe W, Rohman M, Breer P, Wyshak G, Santangelo S, Magnuson E. Alcohol use and abuse in random samples of physicians and medical students. Am J Public Health 1991; 81: 177-82.

21. Aristeiguieta C. Substance abuse, mental illness and medical students: The role of the americans with disabilities act. JAMA 1998; 279: 80-1.

22. PÄrna K, Rahu K, Rahu M. Smoking habits and attitudes towards smoking among Estonian physicians. Public Health 2005; 119: 390-9.

23. Yan J, Xiao S, Ouyang D, Jiang D, He C, Yi S. Smoking behavior, knowledge, attitudes and practice among health care providers in Changsha city, China. Nicotine Tob Res 2008; 10: 737-44.

24. GoldBerg DP. The detection of psychiatric illness by questionnaire. Maudsley Monographs, 21, London, Oxford University Press, 1972.

25. Trucco M, Campusano M, Larraín S. Un cuestionario para detectar desórdenes emocionales. Un estudio de validación preliminar. Rev Chil Neuro-Psiquiatr 1979; 17: 20-5.

26. Benítez C, Quintero J, Torres R. Prevalencia de riesgo de trastornos psiquiátricos en estudiantes de pregrado de la Escuela de Medicina de la P. Universidad Católica de Chile. Rev Méd Chile 2001; 19: 173-8.

27. Organización Panamericana de la Salud. La Salud en las Américas. Publicación Científica y Técnica № 587. Vol. 1, Ed. 2002; 216. 